\title{
Cardiorespiratory Responses during Aquatic Treadmill Exercise and Land Treadmill Exercise in Adults with Diabetes
}

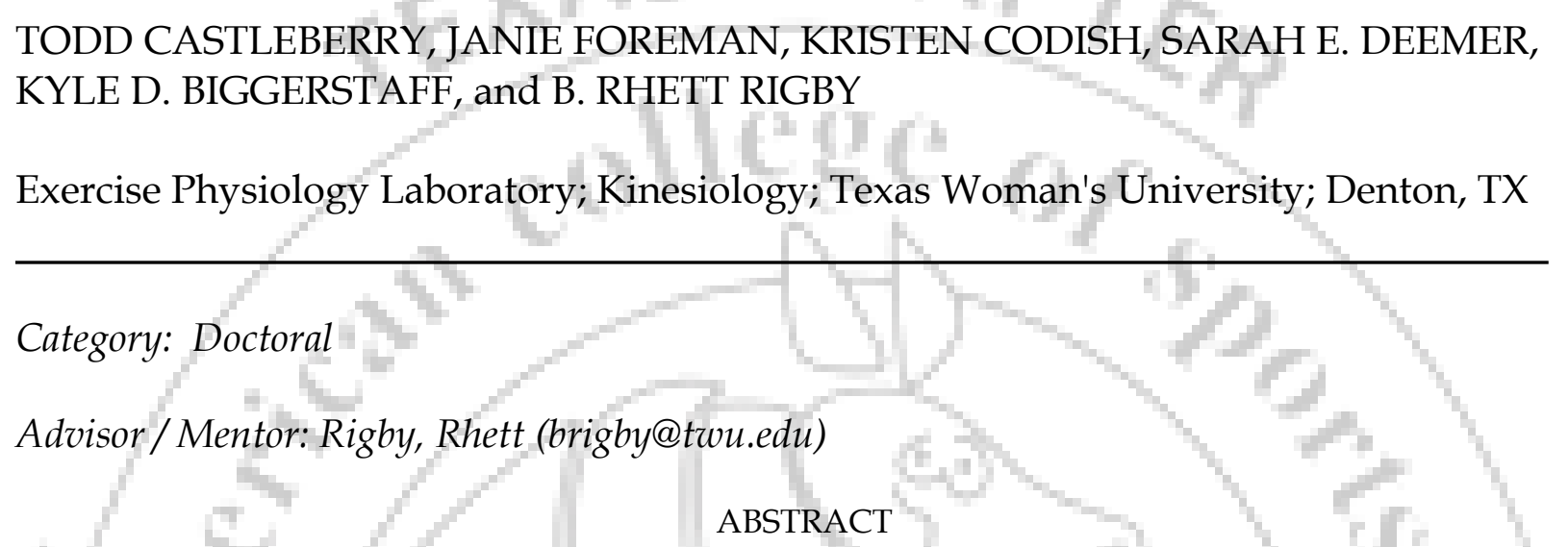

The purpose of this study was to compare the effect of aquatic treadmill (ATM) exercise to land treadmill (LTM) exercise in adults with type 2 diabetes. Five participants with type 2 diabetes (T2D group; 4 females, 1 male; age $=51 \pm 6$ years; height $=170 \pm 7 \mathrm{~cm}$; weight $=96 \pm 24 \mathrm{~kg}$; body fat $=31.6 \pm 2.2 \%$ ) and five participants without type 2 diabetes (control group; 4 females, 1 male; age $=51 \pm 6$ years; height $=170 \pm 6 \mathrm{~cm}$; weight $=71 \pm 15 \mathrm{~kg}$; body fat $=26.8 \pm 4.6 \%$ ) completed the study. Protocols for both ATM exercise and LTM exercise began at $2 \mathrm{mph}$ with $0 \%$ grade and increased by $1 \mathrm{mph}$ after 5 minutes at each stage. Termination occurred after participants completed the protocol or reached $85 \%$ of heart rate reserve. Heart rate, absolute and relative $\mathrm{VO}_{2}$, and systolic and diastolic blood pressure were measured at rest and during steady-state exercise at each intensity. Mean arterial pressure (MAP) was calculated. A $2 \times 2 \times 3$ Mixed Factorial ANOVA and Bonferroni post hoc test with a significance level of .0125 were used. There was a significant difference $(p<.0125)$ in all measures with an increase in intensity for each mode of exercise. Heart rate response was significantly different at $2 \mathrm{mph}$ and $4 \mathrm{mph}$ between LTM exercise and ATM exercise for those with type 2 diabetes (LTM@ 2 mph: 101 \pm 12 bpm vs. ATM @ 2 mph: $92 \pm 8$ bpm, p<.0125; LTM@ 4 mph: $140 \pm 18$ bpm vs. ATM @ 4 mph: $123 \pm 12$ bpm, p<.0125) and those without type 2 diabetes (LTM @ 2 mph: $91 \pm 10$ bpm vs. ATM @ 2 mph: 82 \pm 10 bpm, p<.0125; LTM @ 4 mph: 125 \pm 15 bpm vs. ATM @ $4 \mathrm{mph}: 113 \pm 12 \mathrm{bpm}, \mathrm{p}<.0125)$. There was a significant difference between the relative $\mathrm{VO}_{2}$ of the two groups at $4 \mathrm{mph}$ while performing the land treadmill exercise (T2D: $14.1 \pm 1.4 \mathrm{ml} / \mathrm{kg} / \mathrm{min}$ vs. control: $18.4 \pm 1.6 \mathrm{ml} / \mathrm{kg} / \mathrm{min}, \mathrm{p}<.0125)$. There was no difference in absolute $\mathrm{VO}_{2}$ between participant groups or modes of exercise. Those with type 2 diabetes had an increased MAP versus those without type 2 diabetes while performing the land treadmill exercise at $2 \mathrm{mph}$ (T2D: $93 \pm 3 \mathrm{mmHg}$ vs. control: $81 \pm 5 \mathrm{mmHg}$, $\mathrm{p}<.0125)$. Although there is some evidence for the varying effects of ATM and LTM exercise when comparing those with type 2 diabetes and those without type 2 diabetes, heart rate, $\mathrm{VO}_{2}$, and MAP respond similarly in both groups during ATM and LTM exercise at most treadmill speeds.

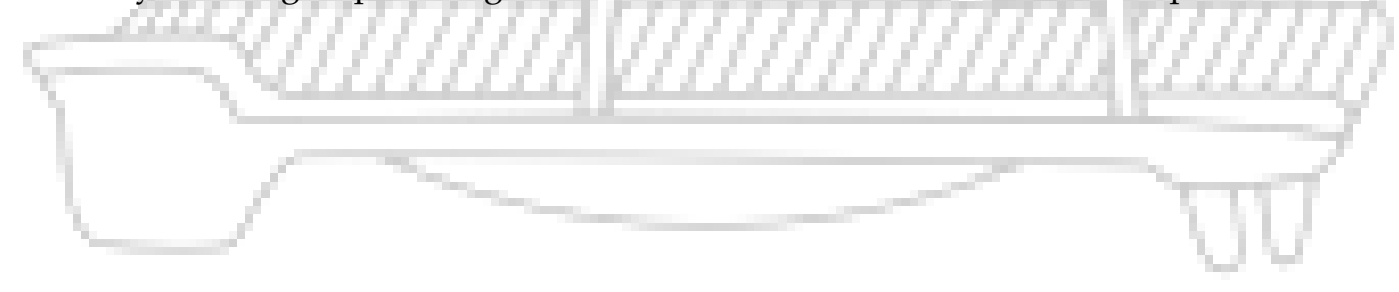

